\title{
Post-Traumatic Narcolepsy Associated with Thalamic/Hypothalamic Injury
}

\author{
Kikyoung Yi, MD¹, Seockhoon Chung, MD, PhD'1, Boram Park, MD¹, Jong Sung Kim, MD, PhD² \\ ${ }^{1}$ Departments of Psychiatry, ${ }^{2}$ Neurology, University of Ulsan College of Medicine, Asan Medical Center, Seoul, Korea
}

The hypothalamus is an important structure that regulates sleep via hypocretin neurotransmission. Central nervous system disorders such as tumors and vascular legions involving the hypothalamus can cause secondary narcolepsy. In addition, brain trauma can contribute to post-traumatic narcolepsy despite lack of any definite brain lesion. Here, we present a case of a 37-year-old man suffering from a hypothalamus-to-thalamus hemorrhage after a traffic accident. After this trauma, he suffered from excessive daytime sleepiness and was diagnosed with post-traumatic narcolepsy by polysomnography and multiple sleep latency tests. He was positive for human leukocyte antigen (HLA)-DQB1*03:01 and HLA-DQB1*06:01 antigens.

Sleep Med Res 2015;6(2):81-83

Key Words Narcolepsy, Brain hemorrhage, Traumatic, HLA-DQB1*0601.

Received: January 22, 2015

Revised: February 6, 2015

Accepted: February 10, 2015

Correspondence

Seockhoon Chung, MD, $\mathrm{PhD}$

Department of Psychiatry,

University of Ulsan College of Medicine,

Asan Medical Center,

88 Olympic-ro 43-gil, Songpa-gu,

Seoul 05505, Korea

Tel $+82-2-3010-3411$

Fax +82-2-485-8381

E-mail schung@amc.seoul.kr

\section{INTRODUCTION}

Narcolepsy is now suggested as an autoimmune disorder. ${ }^{1}$ Autoimmune-mediated loss of hypocretin-producing neurons in the hypothalamus and resultant decreased hypocretin levels in the cerebrospinal fluid might be important to the etiology of narcolepsy. Recently, secondary causes such as infection, trauma, tumors, and H1N1 flu vaccination have been identified as causes or triggers of narcolepsy and excessive daytime sleepiness. ${ }^{2-4}$ Since the first description of post-traumatic narcolepsy, approximately 20 such patients have been reported. ${ }^{5}$ However, studies showing the location of brain damage are rare. ${ }^{6}$ Here, we report a patient who developed polysomnography-confirmed post-traumatic narcolepsy after traumatic hypothalamic hemorrhagic injury.

\section{CASE REPORT}

A 37-year-old man was referred to our hospital due to excessive daytime somnolence and with a history of a trauma resulting from a car accident, in which he lost consciousness, 6 months prior. He had developed daytime sleepiness following the accident that impaired his daytime activities. History taking revealed that before the accident, he had mild daytime sleepiness but no functional impairment and a relatively regular sleep pattern (asleep at 11 pm-12 am and waking up at 6 am). Middle-of-the-night awakenings were rare, and he had no history of snoring, sleep apnea, or restless legs syndrome. During the day, he would nod off and become tired, with excessive sleepiness lasting nearly all day. He had no history of cataplexy, hypnogogic hallucinations, or sleep paralysis. He did not consume alcohol or tobacco and he drank three cups of coffee a day. He didn't show mood or psychotic symptoms when he was interviewed by a psychiatrist (S.C.). He scored 15 on the Epworth Sleepiness Scale (ESS) when he underwent a nocturnal polysomnography. 


\section{Evaluations}

Brain magnetic resonance imaging (MRI) performed 2 weeks after the trauma in this patient revealed a hemorrhage in the right thalamus and midbrain (Fig. 1A). In addition, a diffuse axonal injury associated with petechial hemorrhages was noted in the bilateral periventricular white matter. A small amount of subacute stage of subdural hemorrhage was also found in the left cerebral convexity. An overnight polysomnography study showed a total sleep time of 429.5 minutes with normal sleep efficiency at $99 \%$, a short sleep latency at 0.5 minutes, and patient showed sleep-onset rapid eye movement period in the nocturnal polysomnography with rapid eye movement latency of 2 minutes. Sleep stage scoring revealed increases in stage R (N1: $8 \%, \mathrm{~N} 2$ : $41.2 \%$, N3: $18.6 \%$, R: $32.1 \%$ ) compared to normal R stage of 20$25 \%{ }^{7}$ A sleep study showed an apnea/hypopnea index of 1.4/ hour, a periodic leg movement index of 4.9/hour, and a spontaneous arousal index of 5.3/hour.

The Multiple Sleep Latency Test of 5 naps indicated a mean sleep latency of 2.3 minutes and 4 sleep onset REM periods. He was positive for both human leukocyte antigen (HLA)-DQB1 *03:01 and HLA-DQB1*06:01 antigens.

\section{Progress}

Based on the findings from the sleep studies, he was diagnosed with narcolepsy without cataplexy, $200 \mathrm{mg}$ of modafinil was administered. A follow-up MRI, at 7 months after car accident, showed a resolving hematoma in the thalamic-hypothalamic area (Fig. 1B). The patient's excessive daytime somnolence significantly improved, and we discontinued modafinil. Despite lack of modafinil, his ESS score was rated as 5 .

\section{DISCUSSION}

Although traumatic narcolepsy was first reported more than 70 years ago, distinction from other sleep disorders such as sleep apnea or post-traumatic hypersomnia has remained unclear. ${ }^{5}$ About 20 cases were reported of post-traumatic narcolepsy with heterogeneous manifestations from clinical presentation, severity of injury, symptoms onset time, and HLA typing. ${ }^{6}$ Narcolepsy can occur after hypothalamic lesions that are related to hypocretin neurotransmission. ${ }^{2}$ In our current case, obvious injury of the hypothalamus was observed, and the patient's symptoms gradually resolved over time with improvements in his narcolepsy symptoms. Most post-traumatic narcolepsy patients experience mild to moderate head injury but with no specific brain injury area determined. ${ }^{6}$ There has been only one previously reported case of post-traumatic narcolepsy with a documented brain lesion and surgical treatment. ${ }^{8}$ While the etiology of post-traumatic narcolepsy remains unknown, blunt injury may cause hypothalamic injury leading to an impaired hypocretin system. ${ }^{6}$ In addition, a previous study has revealed loss of hypocretin neurons following post-traumatic brain injury on postmortem examination. ${ }^{9}$

Although HLA typing is not a diagnostic criterion according to the International Classification of Sleep Disorders 2nd Edition, HLA-DQB1 ${ }^{*} 06: 02$ positivity is considered to be supportive of narcolepsy with cataplexy. ${ }^{10}$ In our current case, the patient was negative for HLA-DQB1*06:02 and positive for HLADQB1*06:01 and HLA-DQB1*03:01. HLA-DQB1*06:01 is regarded as a protective allele for narcolepsy, whereas HLADQB1*03:01 is regarded as a weak predisposing allele. ${ }^{11}$ In a previously reported post-traumatic narcolepsy case, such HLA typing was not observed, ${ }^{6}$ and further studies are thus needed on the relationship between HLA and narcolepsy.

This case report describes narcolepsy after a traumatic hem-

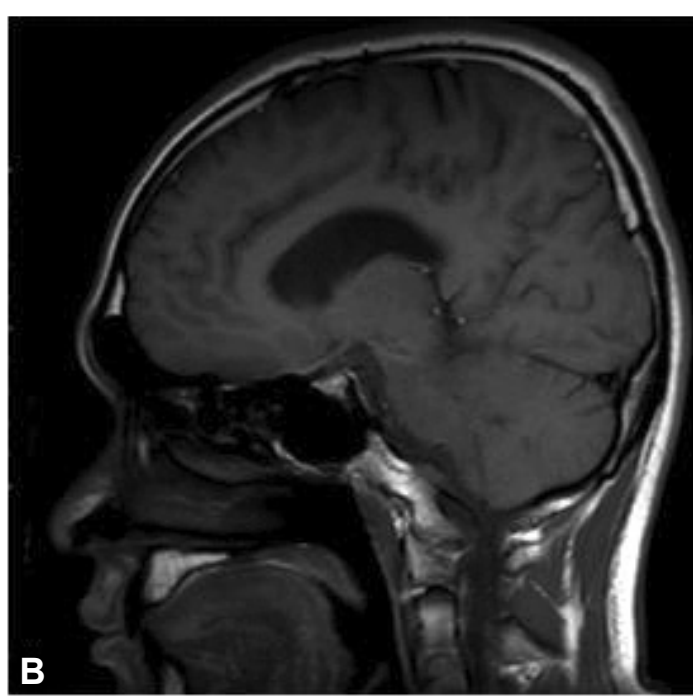

Fig. 1. The sagittal view of the brain MRI of patient with post-traumatic narcolepsy. A: Hemorrhage in the right hypothalamus and right thalamus. B: Resolved hemorrhage in the right hypothalamus and right thalamus.

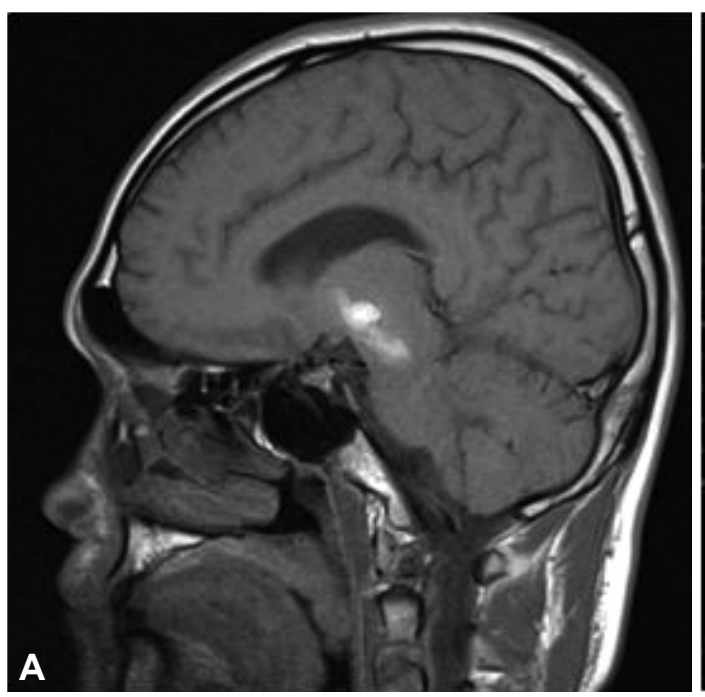


orrhage of the brain, with symptoms resolving with improvement of the brain hemorrhage.

\section{Conflicts of Interest}

The authors have no financial conflicts of interest.

\section{REFERENCES}

1. Mignot E, Hayduk R, Black J, Grumet FC, Guilleminault C. HLA DQB1*0602 is associated with cataplexy in 509 narcoleptic patients. Sleep 1997;20:1012-20.

2. Kanbayashi T, Sagawa Y, Takemura F, Ito SU, Tsutsui K, Hishikawa Y, et al. The pathophysiologic basis of secondary narcolepsy and hypersomnia. Curr Neurol Neurosci Rep 2011;11:235-41.

3. Dauvilliers Y, Montplaisir J, Cochen V, Desautels A, Einen M, Lin L, et al. Post-H1N1 narcolepsy-cataplexy. Sleep 2010;33:1428-30.

4. Castriotta RJ, Wilde MC, Lai JM, Atanasov S, Masel BE, Kuna ST.
Prevalence and consequences of sleep disorders in traumatic brain injury. J Clin Sleep Med 2007;3:349-56.

5. Gill AW. Idiopathic and traumatic narcolepsy. Lancet 1941;237:474-6.

6. Ebrahim IO, Peacock KW, Williams AJ. Posttraumatic narcolepsy-two case reports and a mini review. J Clin Sleep Med 2005;1:153-6.

7. Carskadon MA, Dement WC. Principles and practice of sleep medicine. 5th ed. St. Louis: Elsevier Saunders 2011;16-26.

8. Castriotta RJ, Lai JM. Sleep disorders associated with traumatic brain injury. Arch Phys Med Rehabil 2001;82:1403-6.

9. Baumann CR, Bassetti CL, Valko PO, Haybaeck J, Keller M, Clark E, et al. Loss of hypocretin (orexin) neurons with traumatic brain injury. Ann Neurol 2009;66:555-9.

10. Leschziner G. Narcolepsy: a clinical review. Pract Neurol 2014;14:323-31.

11. Hong SC, Lin L, Lo B, Jeong JH, Shin YK, Kim SY, et al. DQB1*0301 and DQB1*0601 modulate narcolepsy susceptibility in Koreans. Hum Immunol 2007;68:59-68. 